\title{
ON THE INFINITE-HORIZON PROBABILITY OF (NON)RUIN FOR INTEGER-VALUED CLAIMS
}

\author{
ZVETAN G. IGNATOV, * Sofia University 'St Kliment Ohridski' \\ VLADIMIR K. KAISHEV, ${ }^{* *}$ City University, London
}

\begin{abstract}
We consider a compound Poisson process whose jumps are modelled as a sequence of positive, integer-valued, dependent random variables, $W_{1}, W_{2}, \ldots$, viewed as insurance claim amounts. The number of points up to time $t$ of the stationary Poisson process which models the claim arrivals is assumed to be independent of $W_{1}, W_{2}, \ldots$ The premium income to the insurance company is represented by a nondecreasing, nonnegative, realvalued function $h(t)$ on $[0, \infty)$ such that $\lim _{t \rightarrow \infty} h(t)=\infty$. The function $h(t)$ is interpreted as an upper boundary. The probability that the trajectory of such a compound Poisson process will not cross the upper boundary in infinite time is known as the infinitehorizon nonruin probability. Our main result in this paper is an explicit expression for the probability of infinite-horizon nonruin, assuming that certain conditions on the premiumincome function, $h(t)$, and the joint distribution of the claim amount random variables, $W_{1}, W_{2}, \ldots$, hold. We have also considered the classical ruin probability model, in which $W_{1}, W_{2}, \ldots$ are assumed to be independent, identically distributed random variables and we let $h(t)=u+c t$. For this model we give a formula for the nonruin probability which is a special case of our main result. This formula is shown to coincide with the infinite-horizon nonruin probability formulae of Picard and Lefèvre (2001), Gerber (1988), (1989), and Shiu (1987), (1989).
\end{abstract}

Keywords: Infinite-time (non)ruin probability; first crossing of an upper boundary problem; risk process; Poisson claim arrival; dependent claim amounts

2000 Mathematics Subject Classification: Primary 60K30

Secondary 60K 99

\section{Introduction}

To introduce our ruin probability model, we will consider a compound Poisson process

$$
S_{t}=\sum_{i=1}^{N_{t}} W_{i},
$$

where $W_{1}, W_{2}, \ldots$ is a sequence of positive, integer-valued, dependent random variables defined on the probability space $(\Omega, g, \mathrm{P})$ and $N_{t}$ denotes the number of points of the Poisson process up to time $t\left(S_{t}=0\right.$ when $\left.N_{t}=0\right)$. We assume that the process $N_{t}$ is independent of the random variables $W_{1}, W_{2}, \ldots$ Consider an upper barrier given by the nondecreasing, nonnegative, real function $h(t)$ on $[0, \infty)$ such that $\lim _{t \rightarrow \infty} h(t)=\infty$ and $h^{-1}(t)=\inf \{y: h(y) \geq t\}$. Define

Received 29 June 2005; revision received 27 November 2005.

* Postal address: Faculty of Economics and Business Administration, Sofia University 'St Kliment Ohridski', 125 Tsarigradsko Shosse Boulevard, bl. 3, Sofia 1113, Bulgaria.

** Postal address: Faculty of Actuarial Science and Statistics, Cass Business School, City University, 106 Bunhill Row, London EC1Y 8TZ, UK. Email address: v.kaishev@city.ac.uk 
the process $R_{t}=h(t)-S_{t}$ and denote by $T$ the time of the first crossing of the trajectory $t \mapsto S_{t}$ and the boundary $t \mapsto h(t)$, i.e.

$$
T:=\inf \left\{t: t>0, R_{t}<0\right\} .
$$

Let us consider the finite time interval $[0, x], x>0$, and denote by $\mathrm{P}(T>x)$ and $\mathrm{P}(T=\infty)$ the probabilities that the trajectory $t \mapsto S_{t}$ will not cross the boundary $t \mapsto h(t)$ in time $x$ and in infinite time, respectively.

The problem of finding such probabilities is known as the first crossing of an upper boundary problem. In the context of insurance mathematics, such models are referred to as ruin probability models, due to the following straightforward interpretation. The random variables $W_{1}, W_{2}, \ldots$ are assumed to model the amounts of consecutive claims arriving at an insurance company according to a stationary Poisson point process $N_{t}$ with intensity $\lambda$. The premium income of the company up to time $t$ is represented by the upper barrier function $h(t), S_{t}$ models the aggregate claim amount at time $t$, and $R_{t}$ is interpreted as the company's risk (surplus) process at time $t$. The probabilities $\mathrm{P}(T>x)$ and $\mathrm{P}(T=\infty)$ are then respectively viewed as the finiteand infinite-horizon nonruin probabilities. Models of this type have been widely studied, in the context of both applied probability and risk theory, and comprise the subject of ruin theory. The literature on ruin theory is extensive. We refer the interested reader to the monographs of Gerber (1979), Asmussen (2000), and Grandel (1991) for an introduction to the subject and its more recent developments. In what follows we will use well-established ruin theory terminology, which will not prevent the reader from following the probabilistic arguments used throughout the paper.

The probability of nonruin in finite time, $\mathrm{P}(T>x)$ in the model introduced above, has been explored recently by Ignatov and Kaishev (2000). They showed that $\mathrm{P}(T>x)$ can be expressed as

$$
\mathrm{P}(T>x)=\sum_{w_{1} \geq 1, \ldots, w_{n} \geq 1} P_{w_{1}, \ldots, w_{n}} \mathrm{P}\left(T>x \mid W_{1}=w_{1}, \ldots, W_{n}=w_{n}\right),
$$

where $n=[h(x)]+1([h(x)]$ denoting the integer part of $h(x))$ and

$$
\mathrm{P}\left(W_{1}=w_{1}, \ldots, W_{i}=w_{i}\right)=P_{w_{1}, \ldots, w_{i}}
$$

is the joint distribution of $W_{1}, W_{2}, \ldots$, with $w_{i} \geq 1, i=1,2, \ldots$ The conditional nonruin probability is

$$
\begin{aligned}
\mathrm{P}(T & \left.>x \mid W_{1}=w_{1}, \ldots, W_{n}=w_{n}\right) \\
& =\mathrm{P}\left(\bigcap_{i=1}^{k-1}\left\{\tau_{1}+\cdots+\tau_{i}>h^{-1}\left(w_{1}+\cdots+w_{i}\right)\right\} \cap\left\{\tau_{1}+\cdots+\tau_{k} \geq x\right\}\right) \\
& =\mathrm{e}^{-\lambda x} \sum_{i=0}^{k-1}(-1)^{i} b_{i}\left(\lambda h^{-1}\left(w_{1}\right), \ldots, \lambda h^{-1}\left(w_{1}+\cdots+w_{i}\right)\right) \sum_{m=0}^{k-i-1} \frac{(\lambda x)^{m}}{m !} .
\end{aligned}
$$

The natural number $k \equiv k\left(w_{1}, \ldots, w_{n}\right), 1 \leq k \leq n$, in (1) is such that

$$
\begin{aligned}
w_{1}+\cdots+w_{k-1} & \leq n-1, \\
w_{1}+\cdots+w_{k} & \geq n,
\end{aligned}
$$


i.e. $k$ depends on $w_{1}, \ldots, w_{n}$. The interarrival times, $\tau_{1}, \tau_{2}, \ldots$, of the Poisson process $N_{t}$ are random variables independent and identically exponentially distributed with parameter $\lambda$. In (1),

$$
b_{i}\left(v_{1}, v_{2}, \ldots, v_{i}\right)=\operatorname{det}\left(\begin{array}{cccccc}
v_{1} & 1 & 0 & \cdots & 0 & 0 \\
\frac{v_{2}^{2}}{2 !} & v_{2} & 1 & \cdots & 0 & 0 \\
\vdots & \vdots & \vdots & \ddots & \vdots & \vdots \\
\frac{v_{i-1}^{i-1}}{(i-1) !} & \frac{v_{i-1}^{i-2}}{(i-2) !} & \frac{v_{i-1}^{i-3}}{(i-3) !} & \cdots & v_{i-1} & 1 \\
\frac{v_{i}^{i}}{i !} & \frac{v_{i}^{i-1}}{(i-1) !} & \frac{v_{i}^{i-2}}{(i-2) !} & \cdots & \frac{v_{i}^{2}}{2 !} & \\
v_{i}
\end{array}\right)
$$

where $v_{i}=\lambda h^{-1}\left(w_{1}+\cdots+w_{i}\right), i=1,2, \ldots$ In the special case with $i=0$, we have $b_{0} \equiv 1$.

Further refinement of the above result in the form of an exact and computationally appealing finite-time nonruin probability formula can be found in Ignatov et al. (2001).

In this paper we will be interested in the probability of infinite-horizon nonruin, $\mathrm{P}(T=\infty)$. In the special case of independent and identically distributed claim amounts $W_{i}$, and under some other hypotheses, by keeping the premium-income function $h(t)$ 'close' to a linear function of $t$ some very elegant expressions for the infinite-horizon probability of ruin have been derived by Picard and Lefèvre (2001). Two remarkable formulae for the latter probability in the classical ruin probability model, when the premium income is linear, have been derived by Gerber (1988), (1989) and Shiu (1987), (1989). Our purpose here will be to give a new, compact formula for the probability of infinite-horizon nonruin in our more general model of possibly dependent claim amounts, assuming that certain conditions on the function $h(t)$ and the joint distribution of the random variables $W_{1}, W_{2}, \ldots$ are satisfied.

The paper is structured as follows. Section 1 is a brief introduction to the ruin probability model, in which we refer to a finite-time ruin probability formula given by Ignatov and Kaishev (2000). We use it in Section 2 to prove our main result, stated as Theorem 1, which gives an explicit expression for $\mathrm{P}(T=\infty)$. In Section 3, for the classical ruin probability model we give a formula for the nonruin probability which is a special case of our main result, (8). This formula is shown to coincide with the nonruin probability formulae of Picard and Lefèvre (2001), Gerber (1988), (1989), and Shiu (1987), (1989). Some auxiliary results are given in Appendix A.

\section{A formula for the probability of infinite (non)ruin}

To formulate and prove our main result, given in Theorem 1, we will need the following two lemmas.

Lemma 1. Let $w_{1}, w_{2}, \ldots$ be a sequence of natural numbers $\left(w_{i} \geq 1, i=1,2, \ldots\right)$ and let $h(x)$ be a nondecreasing real function defined on $[0, \infty)$ such that $\lim _{x \rightarrow \infty} h(x)=\infty$. Also, let the sequence $\left(q-\lambda h^{-1}\left(w_{1}+\cdots+w_{q}\right)\right)\left(\lambda h^{-1}\left(w_{1}+\cdots+w_{q}\right)\right)^{-1 / 2}, q=1,2, \ldots$, have a limit $a \in[-\infty, \infty]$, i.e.

$$
\lim _{q \rightarrow \infty} \frac{q-\lambda h^{-1}\left(w_{1}+\cdots+w_{q}\right)}{\sqrt{\lambda h^{-1}\left(w_{1}+\cdots+w_{q}\right)}}=a .
$$


Then

$$
\lim _{q \rightarrow \infty} \mathrm{e}^{-\lambda h^{-1}\left(w_{1}+\cdots+w_{q}\right)} \sum_{m=0}^{q-j} \frac{\left(\lambda h^{-1}\left(w_{1}+\cdots+w_{q}\right)\right)^{m}}{m !}=\frac{1}{\sqrt{2 \pi}} \int_{-\infty}^{a} \mathrm{e}^{-x^{2} / 2} \mathrm{~d} x
$$

for every integer $j$.

Remark 1. Several examples can be given in which condition (3) of Lemma 1 is fulfilled. Let $W_{1}>0, W_{2}>0, \ldots$ be a sequence of random variables for which the strong law of large numbers holds, i.e. for which there exists a set $\tilde{H} \in g, \mathrm{P}(\tilde{H})=1$, and a suitable constant $d \geq 0$ such that, with $w_{1}=W_{1}(\omega), \ldots, w_{q}=W_{q}(\omega), \lim _{q \rightarrow \infty}\left(w_{1}+\cdots+w_{q}\right) / q=d$ for all $\omega \in \tilde{H}$. Then, for $h(t)=u+c t, c>\lambda d, \lambda>0$, (3) is fulfilled with $a=\infty$. The proof of this statement is rather standard and is thus omitted. In particular, when $W_{1}, W_{2}, \ldots$ are integer-valued, independent, identically distributed random variables possessing all moments, $d=\mathrm{E}\left(W_{1}\right)$ and we are in the classical ruin probability model. Keeping the latter assumptions on $W_{1}, W_{2}, \ldots$, if $h(t)=t^{\alpha}, t \geq 0, \alpha \geq 1$, then (3) holds with $a=\infty$, since $h^{-1}(t)=t^{1 / \alpha}$, i.e. $h^{-1}(t)=t^{\beta}, 0<\beta \leq 1$, and

$$
\frac{q-\lambda\left(w_{1}+\cdots+w_{q}\right)^{\beta}}{\sqrt{\lambda\left(w_{1}+\cdots+w_{q}\right)^{\beta}}} \rightarrow \infty \quad \text { as } q \rightarrow \infty .
$$

Furthermore, if $h(t)=\ln (1+t), t>0$, we have $h^{-1}(t)=\mathrm{e}^{t}-1$ and it can be shown that

$$
\frac{q-\lambda\left(\mathrm{e}^{w_{1}+\cdots+w_{q}}-1\right)}{\sqrt{\lambda\left(\mathrm{e}^{w_{1}+\cdots+w_{q}}-1\right)}} \rightarrow-\infty \quad \text { as } q \rightarrow \infty
$$

i.e. (3) holds with $a=-\infty$.

For convenience, let us denote the limit in (4) by

$$
\gamma=\frac{1}{\sqrt{2 \pi}} \int_{-\infty}^{a} \mathrm{e}^{-x^{2} / 2} \mathrm{~d} x
$$

Under some appropriate conditions, the following lemma gives an expression for the probability of nonruin, $\mathrm{P}(T=\infty)$, assuming that the individual claim amounts are constants.

Lemma 2. Assume that the assumptions of Lemma 1 hold and that

$$
\mathrm{P}\left(W_{1}=w_{1}, W_{2}=w_{2}, \ldots\right)=1 .
$$

If

$$
\sum_{j=0}^{\infty}(-1)^{j} b_{j}\left(\lambda h^{-1}\left(w_{1}\right), \ldots, \lambda h^{-1}\left(w_{1}+\cdots+w_{j}\right)\right)<\infty
$$

then

$$
\begin{aligned}
\mathrm{P}(T & \left.=\infty \mid W_{1}=w_{1}, W_{2}=w_{2}, \ldots\right) \\
& =\gamma \sum_{j=0}^{\infty}(-1)^{j} b_{j}\left(\lambda h^{-1}\left(w_{1}\right), \ldots, \lambda h^{-1}\left(w_{1}+\cdots+w_{j}\right)\right) .
\end{aligned}
$$


Remark 2. Note that (5) is required to hold because the series it contains may be divergent for some realizations $w_{1}, w_{2}, \ldots$, i.e. the series $\sum_{j=0}^{\infty}(-1)^{j} b_{j}\left(v_{1}, v_{2}, \ldots, v_{j}\right)$ is not always convergent if $v_{1} \leq v_{2} \leq \cdots$ (where $v_{i}=\lambda h^{-1}\left(w_{1}+\cdots+w_{i}\right)$ ). In order to show this, note that $\left|(-1)^{0} b_{0}\right|=1 \times 1=1 \geq 1$ for $j=0$. Let us assume that a similar inequality holds for all $j=1, \ldots, i$, for some appropriate values $v_{1}, v_{2}, \ldots, v_{i}$. Then consider the determinant (recall (2))

$$
b_{i+1}\left(v_{1}, v_{2}, \ldots, v_{i}, x\right) .
$$

If we expand (6) with respect to the last row, we can express it as a polynomial in $x$ of degree $i+1$ of the form

$$
\begin{aligned}
b_{i+1}\left(v_{1}, v_{2}, \ldots, v_{i}, x\right)= & (-1)^{i+1+1} \frac{x^{i+1}}{(i+1) !}+(-1)^{i+1+2} \frac{x^{i}}{i !} b_{1}\left(z_{1}\right)+\cdots \\
& +(-1)^{i+1+i+1} \frac{x^{1}}{1 !} b_{i}\left(z_{1}, \ldots, z_{i}\right) .
\end{aligned}
$$

Since every polynomial converges to $\pm \infty$ as $x \rightarrow \infty$, we can choose a value of $x$ sufficiently large that

$$
\left|b_{i+1}\left(v_{1}, v_{2}, \ldots, v_{i}, x\right)\right| \geq 1 .
$$

Let us choose $x \equiv v_{i+1}$. There then exists a sequence $v_{1}, v_{2}, \ldots$ such that each element of the series $\sum_{j=0}^{\infty}(-1)^{j} b_{j}\left(v_{1}, v_{2}, \ldots, v_{j}\right)$ is greater than or equal to unity in absolute value, i.e. the series is divergent.

To extend the result of Lemma 2 , denote by $D(h)$ the set of all sets $\left\{w_{1}, w_{2}, \ldots\right\}$ such that the conditions of Lemma 1 hold for a fixed $h(x)$ and $a=\infty$. Let

$$
\tilde{D}(h)=\left\{\omega:\left\{W_{1}(\omega), W_{2}(\omega), \ldots\right\} \in D(h)\right\} .
$$

We are now in a position to formulate our main result.

Theorem 1. Let $\mathrm{P}(\tilde{D}(h))=1$ and, for every $\omega \in \tilde{D}(h)$, let

$$
\sum_{j=0}^{\infty}(-1)^{j} b_{j}\left(\lambda h^{-1}\left(W_{1}(\omega)\right), \ldots, \lambda h^{-1}\left(W_{1}(\omega)+\cdots+W_{j}(\omega)\right)\right)<\infty .
$$

Then

$$
\mathrm{P}(T=\infty)=\mathrm{E}\left(\sum_{j=0}^{\infty}(-1)^{j} b_{j}\left(\lambda h^{-1}\left(W_{1}\right), \ldots, \lambda h^{-1}\left(W_{1}+\cdots+W_{j}\right)\right)\right) .
$$

Remark 3. Note that the sequence of random variables

$$
\sum_{j=0}^{q}(-1)^{j} b_{j}\left(\lambda h^{-1}\left(W_{1}\right), \ldots, \lambda h^{-1}\left(W_{1}+\cdots+W_{j}\right)\right), \quad q=0,1,2, \ldots,
$$

is in general not monotone. For example, if $\lambda=1$ and $h(t)=t$ and if $W_{1}(\omega)=\frac{1}{2}, W_{2}(\omega)=\frac{7}{4}$, $W_{4}(\omega)=\frac{19}{16}$, and $W_{5}(\omega)=\frac{29}{32}$, then for $q=0,1,2,3,4,5$ the values of the sequence (9) are respectively equal to $1,0.5,-0.90625,1.24251,-1.64439$, and 2.3864 . Hence, we can not directly interchange the expectation and summation in (8). It is shown by the following corollary of Theorem 1 that this is possible if some (monotonicity) conditions hold for the sequence of random variables (9). 
Corollary 1. Let all multivariate moments of the random variables

$$
h^{-1}\left(W_{1}\right), h^{-1}\left(W_{1}+W_{2}\right), \ldots,
$$

be finite and let the conditions of Theorem 1 hold. If there exists a random variable $\zeta \geq 0$ such that $\mathrm{E}(\zeta)<\infty$ and if

$$
\left|\sum_{j=0}^{q}(-1)^{j} b_{j}\left(\lambda h^{-1}\left(W_{1}(\omega)\right), \ldots, \lambda h^{-1}\left(W_{1}(\omega)+\cdots+W_{j}(\omega)\right)\right)\right| \leq \zeta(\omega)
$$

for all $q \geq 0$ and all $\omega \in \tilde{D}(h)$, then

$$
\mathrm{P}(T=\infty)=\sum_{j=0}^{\infty}(-1)^{j} \mathrm{E}\left(b_{j}\left(\lambda h^{-1}\left(W_{1}\right), \ldots, \lambda h^{-1}\left(W_{1}+\cdots+W_{j}\right)\right)\right) .
$$

Proof of Lemma 1. Assume that $\xi_{1}, \ldots, \xi_{q}$ are independent, identically Poisson-distributed random variables with mean $\lambda h^{-1}\left(w_{1}+\cdots+w_{q}\right) / q$. Then we can write

$$
\begin{aligned}
& \mathrm{e}^{-\lambda h^{-1}\left(w_{1}+\cdots+w_{q}\right)} \sum_{m=0}^{q-j} \frac{\left(\lambda h^{-1}\left(w_{1}+\cdots+w_{q}\right)\right)^{m}}{m !} \\
& =\mathrm{P}\left(\xi_{1}+\cdots+\xi_{q} \leq q-j\right) \\
& \quad=\mathrm{P}\left(\frac{\xi_{1}+\cdots+\xi_{q}-\lambda h^{-1}\left(w_{1}+\cdots+w_{q}\right)}{\sqrt{\lambda h^{-1}\left(w_{1}+\cdots+w_{q}\right)}} \leq \frac{q-j-\lambda h^{-1}\left(w_{1}+\cdots+w_{q}\right)}{\sqrt{\lambda h^{-1}\left(w_{1}+\cdots+w_{q}\right)}}\right) .
\end{aligned}
$$

Applying the central limit theorem, it is not difficult to see that

$$
\begin{aligned}
& \lim _{q \rightarrow \infty} \mathrm{P}\left(\frac{\xi_{1}+\cdots+\xi_{q}-\lambda h^{-1}\left(w_{1}+\cdots+w_{q}\right)}{\sqrt{\lambda h^{-1}\left(w_{1}+\cdots+w_{q}\right)}} \leq \frac{q-j-\lambda h^{-1}\left(w_{1}+\cdots+w_{q}\right)}{\sqrt{\lambda h^{-1}\left(w_{1}+\cdots+w_{q}\right)}}\right) \\
& \quad=\frac{1}{\sqrt{2 \pi}} \int_{-\infty}^{a} \mathrm{e}^{-x^{2} / 2} \mathrm{~d} x .
\end{aligned}
$$

Hence, from (10) and (11), given that condition (3) holds, we recover the assertion of Lemma 1.

Proof of Lemma 2. From the monotone continuity of the probability measure, we have

$$
\begin{aligned}
\mathrm{P}(T & \left.=\infty \mid W_{1}=w_{1}, W_{2}=w_{2}, \ldots\right) \\
& =\mathrm{P}\left(\bigcap_{j=1}^{\infty}\left\{\tau_{1}+\cdots+\tau_{j}>h^{-1}\left(w_{1}+\cdots+w_{j}\right)\right\}\right) \\
& =\lim _{q \rightarrow \infty} \mathrm{P}\left(\bigcap_{j=1}^{q}\left\{\tau_{1}+\cdots+\tau_{j}>h^{-1}\left(w_{1}+\cdots+w_{j}\right)\right\}\right) .
\end{aligned}
$$


From (1), by replacing $x$ with $h^{-1}\left(w_{1}+\cdots+w_{q}\right)$ it is easy to see that $k=q+1$ and

$$
\begin{aligned}
\mathrm{P}\left(\bigcap_{j=1}^{q}\left\{\tau_{1}+\cdots+\tau_{j}>h^{-1}\left(w_{1}+\cdots+w_{j}\right)\right\} \cap\left\{\tau_{1}+\cdots+\tau_{q+1} \geq h^{-1}\left(w_{1}+\cdots+w_{q}\right)\right\}\right) \\
=\mathrm{P}\left(\bigcap_{j=1}^{q}\left\{\tau_{1}+\cdots+\tau_{j}>h^{-1}\left(w_{1}+\cdots+w_{j}\right)\right\}\right) \\
=\mathrm{e}^{-\lambda h^{-1}\left(w_{1}+\cdots+w_{q}\right)} \\
\quad \times \sum_{j=0}^{q}(-1)^{j} b_{j}\left(\lambda h^{-1}\left(w_{1}\right), \ldots, \lambda h^{-1}\left(w_{1}+\cdots+w_{j}\right)\right) \\
\quad \times \sum_{m=0}^{q-j} \frac{\left(\lambda h^{-1}\left(w_{1}+\cdots+w_{q}\right)\right)^{m}}{m !} .
\end{aligned}
$$

We will show now that as $q \uparrow \infty$, the expression on the right-hand side of (13) converges to $\gamma \sum_{\text {Let }}^{\infty}(-1)^{j} b_{j}\left(\lambda h^{-1}\left(w_{1}\right), \ldots, \lambda h^{-1}\left(w_{1}+\cdots+w_{j}\right)\right)$, where $\gamma$ is as defined after Remark 1 .

$$
\begin{gathered}
\beta(j)=(-1)^{j} b_{j}\left(\lambda h^{-1}\left(w_{1}\right), \ldots, \lambda h^{-1}\left(w_{1}+\cdots+w_{j}\right)\right), \\
B_{l}=\sum_{j=0}^{l} \beta(j), l=0, \ldots, \infty, B=\lim _{l \rightarrow \infty} B_{l}, \text { and } \\
\alpha(q, j)=\mathrm{e}^{-\lambda h^{-1}\left(w_{1}+\cdots+w_{q}\right)} \sum_{m=0}^{q-j} \frac{\left(\lambda h^{-1}\left(w_{1}+\cdots+w_{q}\right)\right)^{m}}{m !},
\end{gathered}
$$

where $q=0,1, \ldots, \infty$ and $j$ depends on $q$, i.e. $j=0,1, \ldots, q$. From Lemma 1 , for any nonnegative integer $j$ we have

$$
\lim _{q \rightarrow \infty} \alpha(q, j)=\gamma
$$

From (12) and (13) we have

$$
\mathrm{P}\left(T=\infty \mid W_{1}=w_{1}, W_{2}=w_{2}, \ldots\right)=\lim _{q \rightarrow \infty} \sum_{j=0}^{q} \beta(j) \alpha(q, j) .
$$

Let us choose an arbitrary number, $\varepsilon>0$. Then, in view of (5), there exists a natural number $L_{1}(\varepsilon)$ such that for any $l>L_{1}(\varepsilon)$,

$$
\left|B_{l}-B\right|<\varepsilon .
$$

From (15), in view of (14) and (16), for the same $\varepsilon>0$ there will exist a natural number $L_{2}(\varepsilon)$ such that for any $l>L_{2}(\varepsilon)$,

$$
\left|\mathrm{P}\left(T=\infty \mid W_{1}=w_{1}, W_{2}=w_{2}, \ldots\right)-\sum_{j=0}^{l} \beta(j) \alpha(l, j)\right|<\varepsilon .
$$

Let $L=\max \left(L_{1}(\varepsilon), L_{2}(\varepsilon)\right)$. Then any $l>L$ can be represented as $l=L+m$, where $m$ is a natural number, and

$$
\left|B_{L+m}-B\right|<\varepsilon
$$


and

$$
\left|\mathrm{P}\left(T=\infty \mid W_{1}=w_{1}, W_{2}=w_{2}, \ldots\right)-\sum_{j=0}^{L+m} \beta(j) \alpha(L+m, j)\right|<\varepsilon .
$$

From (17), we can also see that for any $m>1$,

$$
\left|B_{L+m}-B_{L+1}\right|<2 \varepsilon .
$$

From the convergence of $B_{l}$, there exists a constant $G>0$ such that

$$
\left|B_{l}\right|<G, \quad l \geq 0
$$

We have

$$
\begin{aligned}
\mid \mathrm{P}(T= & \left.\infty \mid W_{1}=w_{1}, W_{2}=w_{2}, \ldots\right)-\gamma B \mid \\
= & \mid \mathrm{P}\left(T=\infty \mid W_{1}=w_{1}, W_{2}=w_{2}, \ldots\right)-\sum_{j=0}^{L+m} \beta(j) \alpha(L+m, j) \\
& +\sum_{j=0}^{L+m} \beta(j) \alpha(L+m, j)-\gamma B_{L+m}+\gamma B_{L+m}-\gamma B \mid \\
\leq & \varepsilon+\left|\sum_{j=0}^{L+m} \beta(j)(\alpha(L+m, j)-\gamma)\right|+\gamma \varepsilon .
\end{aligned}
$$

In the inequality in (21) we have used (17), (18), and the definition of $B_{L+m}$. We can rewrite (21) as

$$
\begin{aligned}
\mid \mathrm{P}(T & \left.=\infty \mid W_{1}=w_{1}, W_{2}=w_{2}, \ldots\right)-\gamma B \mid \\
\leq & \varepsilon(1+\gamma)+\left|\sum_{j=0}^{L} \beta(j)(\alpha(L+m, j)-\gamma)\right|+\left|\sum_{j=L+1}^{L+m} \beta(j)(\alpha(L+m, j)-\gamma)\right| \\
\leq & \varepsilon(1+\gamma)+G\{|(\alpha(L+m, 0)-\gamma)|+2|(\alpha(L+m, L)-\gamma)|\} \\
& +\left|\sum_{j=L+1}^{L+m} \beta(j)(\alpha(L+m, j)-\gamma)\right| .
\end{aligned}
$$

In the last inequality in (22) we have used (20) and a result (see, e.g. Fichtenholz (1969), Section 383) which states that, given real sequences $\alpha_{i}$ and $\beta_{i}, i=1,2, \ldots, p$,

$$
\left|\sum_{i=1}^{p} \alpha_{i} \beta_{i}\right| \leq G\left\{\left|\alpha_{1}\right|+2\left|\alpha_{p}\right|\right\}
$$

if $\left|\sum_{i=1}^{s} \beta_{i}\right| \leq G$ for $s=1,2, \ldots, p$ and if $\alpha_{i}, i=1,2, \ldots, p$, is either increasing or decreasing. In our case $\alpha_{i} \equiv \alpha(L+m, i)-\gamma$ and $\beta_{i} \equiv \beta(i), i=0,1, \ldots, L$. Note that the sequence $\alpha_{i}$ is decreasing and that it follows from (20) that $\left|\sum_{i=0}^{s} \beta_{i}\right| \leq G$ for $s=0,1,2, \ldots$ 
From (14), we can choose $m$ to be sufficiently large that $|\alpha(L+m, L)-\gamma|<\varepsilon$ and $|\alpha(L+m, 0)-\gamma|<\varepsilon$. Therefore, from (22) we have

$$
\begin{aligned}
\mid \mathrm{P}(T & \left.=\infty \mid W_{1}=w_{1}, W_{2}=w_{2}, \ldots\right)-\gamma B \mid \\
& \leq \varepsilon(1+\gamma)+G\{\varepsilon+2 \varepsilon\}+\left|\sum_{j=L+1}^{L+m} \beta(j)(\alpha(L+m, j)-\gamma)\right| .
\end{aligned}
$$

Similarly, it can be seen from (19) that $\left|\sum_{j=L+1}^{L+i} \beta(j)\right|<2 \varepsilon$ for $i=1,2, \ldots, m$. Thus, by applying (23) to the sum on the right-hand side of (24) with $\alpha_{i} \equiv(\alpha(L+m, i)-\gamma)$ and $\beta_{i} \equiv \beta(i), i=L+1, \ldots, L+m$, and $G=2 \varepsilon$, we have

$$
\begin{aligned}
& \left|\sum_{j=L+1}^{L+m} \beta(j)(\alpha(L+m, j)-\gamma)\right| \\
& \quad \leq 2 \varepsilon\{|\alpha(L+m, L+1)-\gamma|+2|\alpha(L+m, L+m)-\gamma|\} .
\end{aligned}
$$

From the fact that $\gamma$ and $\alpha(q, j)$ are probabilities, we have $|\alpha(L+m, L+1)-\gamma| \leq 1$ and $|\alpha(L+m, L+m)-\gamma| \leq 1$, and we can rewrite (25) as

$$
\left|\sum_{j=L+1}^{L+m} \beta(j)(\alpha(L+m, j)-\gamma)\right| \leq 2 \varepsilon\{1+2 \times 1\}=6 \varepsilon .
$$

By applying (26) to the sum on the right-hand side of (24), we obtain

$$
\left|\mathrm{P}\left(T=\infty \mid W_{1}=w_{1}, W_{2}=w_{2}, \ldots\right)-\gamma B\right| \leq \varepsilon(1+\gamma)+G\{\varepsilon+2 \varepsilon\}+6 \varepsilon .
$$

Hence, $\mathrm{P}\left(T=\infty \mid W_{1}=w_{1}, W_{2}=w_{2}, \ldots\right)=\gamma B$, which completes the proof of Lemma 2 .

Proof of Theorem 1. From the monotone continuity of the probability measure $\mathrm{P}$ we have, on the one hand,

$$
\mathrm{P}(T=\infty)=\lim _{q \uparrow \infty} \mathrm{P}\left(\bigcap_{j=1}^{q}\left\{\tau_{1}+\cdots+\tau_{j}>h^{-1}\left(W_{1}+\cdots+W_{j}\right)\right\}\right) .
$$

On the other hand, using the notation $A_{q}=\bigcap_{j=1}^{q}\left\{\tau_{1}+\cdots+\tau_{j}>h^{-1}\left(W_{1}+\cdots+W_{j}\right)\right\}$, $q=1,2, \ldots, \infty$, we have

$$
\begin{aligned}
& \mathrm{P}\left(\bigcap_{j=1}^{q}\left\{\tau_{1}+\cdots+\tau_{j}>h^{-1}\left(W_{1}+\cdots+W_{j}\right)\right\}\right) \\
& =\mathrm{E}\left(\mathbf{1}_{A_{q}}\right)=\mathrm{E}\left(\mathrm{E}\left(\mathbf{1}_{A_{q}} \mid W_{1}, \ldots, W_{q}\right)\right) \\
& =\mathrm{E}\left(\mathrm{e}^{-\lambda h^{-1}\left(W_{1}+\cdots+W_{q}\right)} \sum_{j=0}^{q}(-1)^{j} b_{j}\left(\lambda h^{-1}\left(W_{1}\right), \ldots, \lambda h^{-1}\left(W_{1}+\cdots+W_{j}\right)\right)\right. \\
& \left.\quad \times \sum_{m=0}^{q-j} \frac{\left(\lambda h^{-1}\left(W_{1}+\cdots+W_{q}\right)\right)^{m}}{m !}\right) .
\end{aligned}
$$


In the last equality of (27) we have used the fact that the left-hand side of the last equality of (13) coincides with $\mathrm{E}\left(\mathbf{1}_{A_{q}} \mid W_{1}=w_{1}, \ldots, W_{q}=w_{q}\right)$ for every $\omega \in \tilde{D}(h)$ with $W_{1}(\omega)=w_{1}$, $W_{2}(\omega)=w_{2}, \ldots$ Hence, in view of (7), by applying Lemma 2 with $\gamma=1$ we have

$$
\begin{gathered}
\lim _{q \rightarrow \infty} \mathrm{e}^{-\lambda h^{-1}\left(W_{1}+\cdots+W_{q}\right)} \sum_{j=0}^{q}(-1)^{j} b_{j}\left(\lambda h^{-1}\left(W_{1}\right), \ldots, \lambda h^{-1}\left(W_{1}+\cdots+W_{j}\right)\right) \\
\times \sum_{m=0}^{q-j} \frac{\left(\lambda h^{-1}\left(W_{1}+\cdots+W_{q}\right)\right)^{m}}{m !} \\
\quad=\sum_{j=0}^{\infty}(-1)^{j} b_{j}\left(\lambda h^{-1}\left(W_{1}\right), \ldots, \lambda h^{-1}\left(W_{1}+\cdots+W_{j}\right)\right) .
\end{gathered}
$$

Hence, from (27) and (28) we have

$$
\begin{array}{r}
\mathrm{E}\left(\mathbf{1}_{A_{q}} \mid W_{1}, \ldots, W_{q}\right) \rightarrow \sum_{j=0}^{\infty}(-1)^{j} b_{j}\left(\lambda h^{-1}\left(W_{1}\right), \ldots, \lambda h^{-1}\left(W_{1}+\cdots+W_{j}\right)\right) \\
\text { almost surely, as } q \rightarrow \infty .
\end{array}
$$

However, as can be seen from (27), the random variable $\mathrm{E}\left(\mathbf{1}_{A_{q}} \mid W_{1}, \ldots, W_{q}\right)$ has unconditional expectation; hence, we can define its conditional expectation with respect to the sequence of random variables $W_{1}, W_{2}, \ldots$ Since $\mathbf{1}_{A_{q}}$ depends only on $W_{1}, \ldots, W_{q}$, and since the latter are independent of $W_{q+1}, W_{q+2}, \ldots$, we have

$$
\mathrm{E}\left(\mathbf{1}_{A_{q}} \mid W_{1}, \ldots, W_{q}\right)=\mathrm{E}\left(\mathbf{1}_{A_{q}} \mid W_{1}, W_{2}, \ldots\right) .
$$

Since $\mathbf{1}_{A_{q} \downarrow} \downarrow \mathbf{1}_{A_{\infty}}$ as $q \rightarrow \infty$, for any $\omega \in \Omega$, and $\mathrm{E}\left(\mathbf{1}_{A_{q}}\right) \leq 1$ for all $q$, the following equality holds:

$$
\mathrm{E}\left(\mathbf{1}_{A_{q}} \mid W_{1}, W_{2}, \ldots\right) \rightarrow \mathrm{E}\left(\mathbf{1}_{A_{\infty}} \mid W_{1}, W_{2}, \ldots\right) \equiv \mathrm{E}\left(\mathbf{1}_{\{T=\infty\}} \mid W_{1}, W_{2}, \ldots\right) .
$$

From (31), (30), and (29) we have

$$
\begin{aligned}
\mathrm{P}\left(T=\infty \mid W_{1}, W_{2}, \ldots\right) & \equiv \mathrm{E}\left(\mathbf{1}_{A_{\infty}} \mid W_{1}, W_{2}, \ldots\right) \\
& \equiv \sum_{j=0}^{\infty}(-1)^{j} b_{j}\left(\lambda h^{-1}\left(W_{1}\right), \ldots, \lambda h^{-1}\left(W_{1}+\cdots+W_{j}\right)\right) .
\end{aligned}
$$

The assertion of Theorem 1 now follows, since $\mathrm{P}(T=\infty)=\mathrm{E}\left(\mathrm{E}\left(\mathbf{1}_{A_{\infty}} \mid W_{1}, W_{2}, \ldots\right)\right)$.

Proof of Corollary 1. The proof is straightforward and is thus omitted.

\section{On the probability of infinite (non)ruin in the classical model}

In this section we will consider the classical ruin probability model in which $h(t)=u+c t$ and $W_{1}, W_{2}, \ldots$ are assumed to be integer-valued, independent, identically distributed random variables having all moments. The following corollary of Theorem 1 gives an expression for the nonruin probability in this classical case. 
Corollary 2. If $c>\lambda \mathrm{E}\left(W_{1}\right)$ then the nonruin probability in the classical model can be expressed as

$$
\mathrm{P}(T=\infty)=\sum_{j=0}^{\infty}(-1)^{j} \mathrm{E}\left(b_{j}(\lambda / c)\left(W_{1}-u\right)_{+}, \ldots,(\lambda / c)\left(W_{1}+\cdots+W_{j}-u\right)_{+}\right),
$$

where $(z)_{+}=\max (0, z)$.

Proof. We show that the conditions of Lemma 1, Theorem 1, and Corollary 1 hold for this classical ruin probability model, from which (32) follows. However, we will take a more direct approach and establish that the right-hand side of (32) is equal to the right-hand side of the formula

$$
\begin{aligned}
\mathrm{P}(T & =\infty) \\
& =\left(1-\frac{\lambda \mathrm{E}\left(W_{1}\right)}{c}\right) \sum_{j=0}^{\infty} \frac{1}{j !}\left(-\frac{\lambda}{c}\right)^{j} \mathrm{E}\left(\left(u-\sum_{i=1}^{j} W_{i}\right)_{+}^{j} \exp \left(\frac{\lambda}{c}\left(u-\sum_{i=1}^{j} W_{i}\right)\right)\right)
\end{aligned}
$$

(of Gerber (1988), (1989) and Shiu (1987), (1989)), which has been shown by Picard and Lefèvre (2001) to coincide with their Equation (3.1), for $\mathrm{P}(T=\infty)$. Following this approach, let us establish identity (32) for $0 \leq u<1$.

We have

$$
\begin{aligned}
\mathrm{P}(T=\infty) & =\sum_{j=0}^{\infty}(-1)^{j} \mathrm{E}\left(b_{j}\left((\lambda / c)\left(W_{1}-u\right)_{+}, \ldots,(\lambda / c)\left(W_{1}+\cdots+W_{j}-u\right)_{+}\right)\right) \\
& =\sum_{j=0}^{\infty}(-1)^{j}\left(\frac{\lambda}{c}\right)^{j} \mathrm{E}\left(b_{j}\left(W_{1}-u, \ldots, W_{1}+\cdots+W_{j}-u\right)\right) .
\end{aligned}
$$

In the last equality of (34) we have used the fact that $0 \leq u<1$ and $W_{1} \geq 1$.

From (34), by applying Lemma 7 (see Appendix A) with $v=-u$ we have

$$
\begin{aligned}
\mathrm{P}(T=\infty)= & (-1)^{0}\left(\frac{\lambda}{c}\right)^{0} \mathrm{E}(1)+(-1)^{1}\left(\frac{\lambda}{c}\right)^{1} \mathrm{E}\left(W_{1}-u\right) \\
& +\sum_{j=2}^{\infty}(-1)^{j}\left(\frac{\lambda}{c}\right)^{j} \mathrm{E}\left(b_{j}\left(W_{1}-u, \ldots, W_{1}+\cdots+W_{j}-u\right)\right) \\
= & 1-\frac{\lambda}{c}\left(\mathrm{E}\left(W_{1}\right)-u\right)+\sum_{j=2}^{\infty}(-1)^{j}\left(\frac{\lambda}{c}\right)^{j}\left(\frac{(-u)^{j}}{j !}+\frac{(-u)^{j-1}}{(j-1) !} \mathrm{E}\left(W_{1}\right)\right) .
\end{aligned}
$$

After a straightforward transformation of the sum on the right-hand side of (35), for $0 \leq u<1$ we obtain

$$
\mathrm{P}(T=\infty)=\mathrm{e}^{\lambda u / c}\left(1-(\lambda / c) \mathrm{E}\left(W_{1}\right)\right) .
$$

As can be seen, (36) coincides with the expression of the nonruin probability, (33), obtained by Gerber (1988), (1989) and Shiu (1987), (1989). Similar but more complicated derivations lead to the same result for any value of $u \geq 1$. This completes the proof of Corollary 2 . 


\section{Appendix A.}

The main auxiliary result, which is used in proving Corollary 2 is given here as Lemma 7. The latter is proved by means of Lemma 6, which is in turn proved via Lemmas 3 to 5 . We present their proofs first. Let us start by introducing some useful notation. Let $\left(l_{1}, \ldots, l_{k}\right)$ be an ordered set of $k$ fixed, nonnegative integers, and let

$$
\pi=\left(\begin{array}{c}
1,2, \ldots, k \\
\pi_{1}, \pi_{2}, \ldots, \pi_{k}
\end{array}\right)
$$

be a permutation of the numbers $(1,2, \ldots, k)$. Denote by $\mathcal{P}_{k}$ the set of all such permutations. Applying $\pi$ let us permute $\left(l_{1}, \ldots, l_{k}\right)$ as follows:

$$
\pi\left(l_{1}, \ldots, l_{k}\right)=\left(l_{\pi_{1}}, \ldots, l_{\pi_{k}}\right) .
$$

Let us introduce the polynomial

$$
Q_{\left(l_{1}, \ldots, l_{k}\right)}\left(x_{1}, \ldots, x_{k}\right)=\sum_{\pi \in \mathcal{P}_{k}} a_{\pi\left(l_{1}, \ldots, l_{k}\right)} x_{1}^{l_{\pi_{1}}} \ldots x_{k}^{l_{\pi_{k}}},
$$

with real coefficients $a_{\pi\left(l_{1}, \ldots, l_{2}\right)}$. We shall refer to it as a permutational polynomial. Such polynomials may have been studied elsewhere, but the authors were not able to find any relevant references. We give here some auxiliary results related to these polynomials.

Lemma 3. We have

$$
\begin{aligned}
\left(x_{1}+\right. & \left.\cdots+x_{k}\right) Q_{\left(l_{1}, \ldots, l_{k}\right)}\left(x_{1}, \ldots, x_{k}\right) \\
& =Q_{\left(l_{1}+1, \ldots, l_{k}\right)}\left(x_{1}, \ldots, x_{k}\right)+\cdots+Q_{\left(l_{1}, \ldots, l_{k}+1\right)}\left(x_{1}, \ldots, x_{k}\right),
\end{aligned}
$$

where the coefficients of the polynomials on the right-hand side satisfy

$$
a_{\pi\left(l_{1}+1, \ldots, l_{k}\right)}=\cdots=a_{\pi\left(l_{1}, \ldots, l_{k}+1\right)}=a_{\pi\left(l_{1}, \ldots, l_{k}\right)} \text { for all } \pi \in \mathcal{P}_{k} .
$$

Lemma 4. We have

$$
\begin{aligned}
& \left(x_{1}+\cdots+x_{k}\right)^{r} Q_{\left(l_{1}, \ldots, l_{k}\right)}\left(x_{1}, \ldots, x_{k}\right) \\
& \quad=\sum_{\substack{j_{1} \geq 0, \ldots, j_{k} \geq 0 \\
j_{1}+\cdots+j_{k}=r}} \frac{r !}{j_{1} ! \cdots j_{k} !} Q_{\left(l_{1}+j_{1}, \ldots, l_{k}+j_{k}\right)}\left(x_{1}, \ldots, x_{k}\right),
\end{aligned}
$$

assuming that

$$
a_{\pi\left(l_{1}+j_{1}, \ldots, l_{k}+j_{k}\right)}=\cdots=a_{\pi\left(l_{1}, \ldots, l_{k}\right)}
$$

for all integers $j_{1} \geq 0, \ldots, j_{k} \geq 0$ and permutations $\pi \in \mathcal{P}_{k}$.

The proof of Lemma 4 is straightforward and will not be given here.

Let us now make the following definition. We will call the permutational polynomial $Q_{\left(l_{1}, \ldots, l_{k}\right)}\left(x_{1}, \ldots, x_{k}\right)$ a 'contrast' if the sum of its coefficients is equal to 0 , i.e. if

$$
\sum_{\pi \in \mathcal{P}_{k}} a_{\pi\left(l_{1}, \ldots, l_{k}\right)}=0
$$


We can now formulate Lemma 5, which we will need to prove Lemma 6.

Lemma 5. If $Q\left(x_{1}, \ldots, x_{k}\right)$ is a linear combination of contrasts and $Y_{1}, Y_{2}, \ldots$ is a sequence of independent and identically distributed random variables having all moments, then

$$
\mathrm{E}\left(\left(Y_{1}+\cdots+Y_{k}+Y_{k+1}+\cdots+Y_{k+s}\right)^{r} Q\left(Y_{1}, \ldots, Y_{k}\right)\right)=0 .
$$

Proof. Let $Q_{\left(l_{1}, \ldots, l_{k}\right)}\left(x_{1}, \ldots, x_{k}\right)$ be a contrast. For the mean, $\mathrm{E}\left(Q_{\left(l_{1}, \ldots, l_{k}\right)}\left(Y_{1}, \ldots, Y_{k}\right)\right)$, we obtain

$$
\begin{aligned}
\mathrm{E}\left(Q_{\left(l_{1}, \ldots, l_{k}\right)}\left(Y_{1}, \ldots, Y_{k}\right)\right) & =\mathrm{E}\left(\sum_{\pi \in \mathcal{P}_{k}} a_{\pi\left(l_{1}, \ldots, l_{k}\right)} Y_{1}^{l_{\pi_{1}}} \cdots Y_{k}^{l_{\pi_{k}}}\right) \\
& =\sum_{\pi \in \mathcal{P}_{k}} a_{\pi\left(l_{1}, \ldots, l_{k}\right)} \mathrm{E}\left(Y_{1}^{l_{\pi_{1}}}\right) \cdots \mathrm{E}\left(Y_{k}^{l_{\pi_{k}}}\right) \\
& =\sum_{\pi \in \mathcal{P}_{k}} a_{\pi\left(l_{1}, \ldots, l_{k}\right)} \mathrm{E}\left(Y_{1}^{l_{1}}\right) \cdots \mathrm{E}\left(Y_{k}^{l_{k}}\right) \\
& =0 \times \mathrm{E}\left(Y_{1}^{l_{1}}\right) \times \cdots \times \mathrm{E}\left(Y_{k}^{l_{k}}\right)=0 .
\end{aligned}
$$

Let us consider the product $\left(Y_{1}+\cdots+Y_{k}+Y_{k+1}+\cdots+Y_{k+s}\right)^{r} Q_{\left(l_{1}, \ldots, l_{k}\right)}\left(Y_{1}, \ldots, Y_{k}\right)$. For its mean value we have

$$
\begin{aligned}
& \mathrm{E}\left(\left(Y_{1}+\cdots+Y_{k}+Y_{k+1}+\cdots+Y_{k+s}\right)^{r} Q_{\left(l_{1}, \ldots, l_{k}\right)}\left(Y_{1}, \ldots, Y_{k}\right)\right) \\
&= \mathrm{E}\left(\sum_{\nu=0}^{r}\left(\begin{array}{l}
r \\
v
\end{array}\right)\left(Y_{1}+\cdots+Y_{k}\right)^{v}\left(Y_{k+1}+\cdots+Y_{k+s}\right)^{r-v} Q_{\left(l_{1}, \ldots, l_{k}\right)}\left(Y_{1}, \ldots, Y_{k}\right)\right) \\
&= \sum_{\nu=0}^{r}\left(\begin{array}{l}
r \\
v
\end{array}\right) \mathrm{E}\left(\left(Y_{1}+\cdots+Y_{k}\right)^{v} Q_{\left(l_{1}, \ldots, l_{k}\right)}\left(Y_{1}, \ldots, Y_{k}\right)\right) \mathrm{E}\left(\left(Y_{k+1}+\cdots+Y_{k+s}\right)^{r-v}\right) \\
&= \sum_{\nu=0}^{r}\left(\begin{array}{l}
r \\
v
\end{array}\right) \mathrm{E}\left(\left(Y_{k+1}+\cdots+Y_{k+s}\right)^{r-v}\right) \\
& \quad \sum_{\substack{j_{1} \geq 0, \ldots, j_{k} \geq 0 \\
j_{1}+\cdots+j_{k}=r}} \frac{r !}{j_{1} ! \cdots j_{k} !} \mathrm{E}\left(Q_{\left(l_{1}+j_{1}, \ldots, l_{k}+j_{k}\right)}\left(Y_{1}, \ldots, Y_{k}\right)\right),
\end{aligned}
$$

where in the third equality we have used (37) to express

$$
\left(Y_{1}+\cdots+Y_{k}\right)^{v} Q_{\left(l_{1}, \ldots, l_{k}\right)}\left(Y_{1}, \ldots, Y_{k}\right)
$$

The permutational polynomial $Q_{\left(l_{1}+j_{1}, \ldots, l_{k}+j_{k}\right)}\left(x_{1}, \ldots, x_{k}\right)$ is a contrast, since it has the same coefficients as $Q_{\left(l_{1}, \ldots, l_{k}\right)}\left(x_{1}, \ldots, x_{k}\right)$. Hence,

$$
\mathrm{E}\left(Q_{\left(l_{1}+j_{1}, \ldots, l_{k}+j_{k}\right)}\left(Y_{1}, \ldots, Y_{k}\right)\right)=0 \quad \text { for all } j_{1} \geq 0, \ldots, j_{k} \geq 0 \text { with } j_{1}+\cdots+j_{k}=r
$$

and, therefore, $\mathrm{E}\left(\left(Y_{1}+\cdots+Y_{k}+Y_{k+1}+\cdots+Y_{k+s}\right)^{r} Q_{\left(l_{1}, \ldots, l_{k}\right)}\left(Y_{1}, \ldots, Y_{k}\right)\right)=0$. Lemma 5 now follows from the fact that the mean is a linear functional. 
We are now in a position to prove the following lemma.

Lemma 6. For every $k \geq 2$ independent and identically distributed random variables $Y_{1}, \ldots, Y_{k}$ having moments of all orders, we have

$$
\begin{gathered}
\left.\operatorname{E}\left(\begin{array}{cccccc}
Y_{1} & 1 & 0 & \cdots & 0 & 0 \\
\frac{\left(Y_{1}+Y_{2}\right)^{2}}{2 !} & Y_{1}+Y_{2} & 1 & \ldots & 0 & 0 \\
\frac{\left(Y_{1}+Y_{2}+Y_{3}\right)^{3}}{3 !} & \frac{\left(Y_{1}+Y_{2}+Y_{3}\right)^{2}}{2 !} & Y_{1}+Y_{2}+Y_{3} & \cdots & 0 & 0 \\
\vdots & \vdots & \vdots & \ddots & \vdots & \vdots \\
\frac{\left(\sum_{\ell=1}^{k-1} Y_{\ell}\right)^{k-1}}{(k-1) !} & \frac{\left(\sum_{\ell=1}^{k-1} Y_{\ell}\right)^{k-2}}{(k-2) !} & \frac{\left(\sum_{\ell=1}^{k-1} Y_{\ell}\right)^{k-3}}{(k-3) !} & \cdots & \sum_{\ell=1}^{k-1} Y_{\ell} & 1 \\
\frac{\left(\sum_{\ell=1}^{k} Y_{\ell}\right)^{k}}{k !} & \frac{\left(\sum_{\ell=1}^{k} Y_{\ell}\right)^{k-1}}{(k-1) !} & \frac{\left(\sum_{\ell=1}^{k} Y_{\ell}\right)^{k-2}}{(k-2) !} & \cdots & \frac{\left(\sum_{\ell=1}^{k} Y_{\ell}\right)^{2}}{2 !} & \sum_{\ell=1}^{k} Y_{\ell}
\end{array}\right)\right) \\
=0 .
\end{gathered}
$$

Proof. We will use induction on $k=2,3, \ldots$ for the sequence of assumed equalities

$$
\mathrm{E}\left(\left(Y_{1}+\cdots+Y_{k}+Y_{k+1}+\cdots+Y_{k+s}\right)^{r} \operatorname{det} D_{k}\right)=0, \quad \text { for all integers } r \geq 0 \text { and } s \geq 0,
$$

where det $D_{k}$ denotes the determinant in (38).

For $k=2$ we have

$$
\begin{aligned}
& \mathrm{E}\left(\left(Y_{1}+Y_{2}+Y_{2+1}+\cdots+Y_{2+s}\right)^{r}\left(Y_{1}\left(Y_{1}+Y_{2}\right)-\frac{\left(Y_{1}+Y_{2}\right)^{2}}{2}\right)\right) \\
& \quad=\mathrm{E}\left(\left(Y_{1}+Y_{2}+Y_{2+1}+\cdots+Y_{2+s}\right)^{r}\left(\frac{1}{2} Y_{1}^{2}-\frac{1}{2} Y_{2}^{2}\right)\right) \\
& \quad=0 .
\end{aligned}
$$

In the last equality we have used Lemma 5 , since the polynomial $\frac{1}{2} x_{1}^{2}-\frac{1}{2} x_{2}^{2}$ is a contrast, i.e.

$$
\frac{1}{2} x_{1}^{2}-\frac{1}{2} x_{2}^{2} \equiv Q_{(2,0)}\left(x_{1}, x_{2}\right)
$$

where, for the two possible permutations, $\pi^{(1)} \in \mathcal{P}_{2}$ and $\pi^{(2)} \in \mathcal{P}_{2}$, we have

$$
\pi^{(1)}=\left(\begin{array}{ll}
1 & 2 \\
1 & 2
\end{array}\right), \quad a_{\pi^{(1)}(2,0)} \equiv \frac{1}{2} \quad \text { and } \quad \pi^{(2)}=\left(\begin{array}{ll}
1 & 2 \\
2 & 1
\end{array}\right), \quad a_{\pi^{(2)}(2,0)} \equiv-\frac{1}{2} .
$$

Let us assume that for every $j, 2 \leq j \leq k$,

$$
\mathrm{E}\left(\left(Y_{1}+\cdots+Y_{k}+Y_{k+1}+\cdots+Y_{k+s}\right)^{r} \operatorname{det} D_{j}\right)=0 .
$$


For $j=k+1, r=0$, and $s=0$, for $\operatorname{det} D_{k+1}$ we have

$$
\begin{aligned}
& \mathrm{E}\left(\operatorname{det} D_{k+1}\right)=\mathrm{E}(\left(Y_{1}+\cdots+Y_{k+1}\right) \operatorname{det} D_{k}-\frac{\left(Y_{1}+\cdots+Y_{k+1}\right)^{2}}{2} \operatorname{det} D_{k-1}+\cdots \\
& \quad+(-1)^{k-2}\left(Y_{1}+\cdots+Y_{k+1}\right)^{k-1} \operatorname{det} D_{2}+(-1)^{k-1} \frac{\left(Y_{1}+\cdots+Y_{k+1}\right)^{k}}{k !} Y_{1} \\
&\left.\quad+(-1)^{k} \frac{\left(Y_{1}+\cdots+Y_{k+1}\right)^{k+1}}{(k+1) !}\right) \\
&= \frac{(-1)^{k-1}}{k !} \mathrm{E}\left(( Y _ { 1 } + \cdots + Y _ { k + 1 } ) ^ { k } \left(\frac{k}{k+1} Y_{1}+\left(-\frac{1}{k+1}\right) Y_{2}+\cdots\right.\right. \\
&\left.\left.\quad+\left(-\frac{1}{k+1}\right) Y_{k+1}\right)\right) .
\end{aligned}
$$

In the last equality in (39) we have used the induction assumption.

It is not difficult to establish that the polynomial

$$
\frac{k}{k+1} x_{1}-\frac{1}{k+1} x_{2}-\cdots-\frac{1}{k+1} x_{k+1}
$$

is a contrast, i.e.

$$
\frac{k}{k+1} x_{1}-\frac{1}{k+1} x_{2}-\cdots-\frac{1}{k+1} x_{k+1} \equiv Q_{(1,0, \ldots, 0)}\left(x_{1}, \ldots, x_{k+1}\right),
$$

where

$$
\begin{gathered}
\pi^{(i)}=\left(\begin{array}{l}
1,2, \ldots, i-1, i, i+1, \ldots, k+1 \\
i, 2, \ldots, i-1,1, i+1, \ldots, k+1
\end{array}\right), \quad i=1,2, \ldots, k+1, \\
a_{\pi^{(1)}(1,0, \ldots, 0)} \equiv \frac{k}{k+1}, \quad \text { and } \quad a_{\pi^{(i)}(1,0, \ldots, 0)} \equiv-\frac{1}{k+1}, \quad 2 \leq i \leq k+1 .
\end{gathered}
$$

Hence,

$$
\begin{aligned}
& \mathrm{E}\left(\left(Y_{1}+\cdots+Y_{k+1}\right)^{k}\left(\frac{k}{k+1} Y_{1}-\frac{1}{k+1} Y_{2}-\cdots-\frac{1}{k+1} Y_{k+1}\right)\right) \\
& \quad=\mathrm{E}\left[\left(Y_{1}+\cdots+Y_{k+1}\right)^{k} Q_{(1,0, \ldots, 0)}\left(Y_{1}, \ldots, Y_{k}\right)\right] \\
& \quad=0 .
\end{aligned}
$$

In the last equality we have used Lemma 5. Now, for the case of any nonnegative integer values of $r$ and $s$, we have

$$
\begin{aligned}
\mathrm{E}\left(\left(Y_{1}+\cdots+Y_{k}+\cdots+Y_{k+s}\right)^{r} \operatorname{det} D_{k+1}\right) \\
=\mathrm{E}\left(\sum_{\nu=0}^{r}\left(\begin{array}{l}
r \\
v
\end{array}\right)\left(Y_{1}+\cdots+Y_{k+1}\right)^{v}\left(Y_{k+2}+\cdots+Y_{k+s}\right)^{r-v} \operatorname{det} D_{k+1}\right) \\
=\sum_{\nu=0}^{r}\left(\begin{array}{l}
r \\
v
\end{array}\right) \mathrm{E}\left(\left(Y_{k+2}+\cdots+Y_{k+s}\right)^{r-v}\right) \mathrm{E}\left(\left(Y_{1}+\cdots+Y_{k+1}\right)^{v} \operatorname{det} D_{k+1}\right)
\end{aligned}
$$


and, by proceeding as for (39) and applying induction, we obtain

$$
\begin{aligned}
\mathrm{E}\left(\left(Y_{1}\right.\right. & \left.\left.+\cdots+Y_{k}+\cdots+Y_{k+s}\right)^{r} \operatorname{det} D_{k+1}\right) \\
= & \sum_{\nu=0}^{r}\left(\begin{array}{l}
r \\
v
\end{array}\right) \mathrm{E}\left(\left(Y_{k+2}+\cdots+Y_{k+s}\right)^{r-v}\right) \frac{(-1)^{k-1}}{(k) !} \\
& \times \mathrm{E}\left(\left(Y_{1}+\cdots+Y_{k+1}\right)^{v+k}\left(\frac{k}{k+1} Y_{1}-\frac{1}{k+1} Y_{2}-\cdots-\frac{1}{k+1} Y_{k+1}\right)\right) \\
= & \sum_{\nu=0}^{r}\left(\begin{array}{l}
r \\
v
\end{array}\right) \mathrm{E}\left(\left(Y_{k+2}+\cdots+Y_{k+s}\right)^{r-v}\right) \frac{(-1)^{k-1}}{(k) !} \times 0 \\
= & 0 .
\end{aligned}
$$

In the last equality of (41) we have used Lemma 5 and (40). This completes the proof of Lemma 6.

Finally, we apply Lemma 6 in proving the following lemma, which we will need to prove Corollary 2.

Lemma 7. Let $v$ and $W_{1}, W_{2}, \ldots$ be independent random variables having moments of all orders and let $W_{1}, W_{2}, \ldots$ be identically distributed. Then, for $j \geq 2$ we have

$$
\mathrm{E}\left(b_{j}\left(v+W_{1}, \ldots, v+W_{1}+\cdots+W_{j}\right)\right)=\frac{\mathrm{E}\left(v^{j}\right)}{j !}+\frac{\mathrm{E}\left(v^{j-1}\right)}{(j-1) !} \mathrm{E}\left(W_{1}\right) .
$$

Proof. The identity

$$
b_{j}\left(v_{1}+c, \ldots, v_{j}+c\right)=b_{j}\left(v_{1}, \ldots, v_{j}\right)-b_{j}\left(v_{1}, \ldots, v_{j}-c\right), \quad j=1,2, \ldots,
$$

follows easily from the fact that

$$
b_{j}\left(v_{1}+c, \ldots, v_{j}+c\right)=\operatorname{det}\left(\begin{array}{cccc}
v_{1} & 1 & \cdots & 0 \\
\frac{v_{2}^{2}}{2 !} & v_{2} & \cdots & 0 \\
\vdots & \vdots & \ddots & \vdots \\
\frac{v_{j-1}^{j-1}}{(i-1) !} & \frac{v_{j-1}^{j-2}}{(i-2) !} & \cdots & 1 \\
\frac{v_{j}^{j}}{j !}-\frac{(-c)^{j}}{j !} & \frac{v_{j}^{i-1}}{(j-1) !}-\frac{(-c)^{j-1}}{(j-1) !} & \cdots & \frac{v_{j}^{1}}{1 !}-\frac{(-c)^{1}}{1 !}
\end{array}\right)
$$

and well-known properties of determinants.

Applying (42) yields

$$
\begin{aligned}
b_{j}(v & \left.+W_{1}, \ldots, v+W_{1}+\cdots+W_{j}\right) \\
& =b_{j}\left(W_{1}, \ldots, W_{1}+\cdots+W_{j}\right)-b_{j}\left(W_{1}, \ldots, W_{1}+\cdots+W_{j}-v\right) \\
& =b_{j}\left(W_{1}, \ldots, W_{1}+\cdots+W_{j}\right)-\sum_{l=1}^{j}(-1)^{l-1} \frac{(-v)^{l}}{l !} b_{j-l}\left(W_{1}, \ldots, W_{1}+\cdots+W_{j-l}\right),
\end{aligned}
$$


where, in the last equality, we have expanded the determinant with respect to its final row. By taking expectations in (43) and noting the independence of the random variables $v$ and $W_{1}, W_{2}, \ldots$, we obtain

$$
\begin{aligned}
& \mathrm{E}\left(b_{j}\left(v+W_{1}, \ldots, v+W_{1}+\cdots+W_{j}\right)\right) \\
& \quad=\mathrm{E}\left(b_{j}\left(W_{1}, \ldots, W_{1}+\cdots+W_{j}\right)\right)+\sum_{l=1}^{j} \frac{\mathrm{E}\left(v^{l}\right)}{l !} \mathrm{E}\left(b_{j-l}\left(W_{1}, \ldots, W_{1}+\cdots+W_{j-l}\right)\right) .
\end{aligned}
$$

We can simplify (44) by applying Lemma 6 to it. Thus, for $j \geq 2$ we have

$$
\mathrm{E}\left(b_{j}\left(v+W_{1}, \ldots, v+W_{1}+\cdots+W_{j}\right)\right)=\frac{\mathrm{E}\left(v^{j}\right)}{j !}+\frac{\mathrm{E}\left(v^{j-1}\right)}{(j-1) !} \mathrm{E}\left(W_{1}\right),
$$

which completes the proof of Lemma 7.

\section{Acknowledgements}

We would like to thank the anonymous referee for his detailed comments and suggestions, which allowed us to shorten and improve the presentation of the paper. The first author thanks the Faculty of Actuarial Science and Statistics, Cass Business School, City University, London for the support received during his visit, when work on the paper was completed.

\section{References}

Asmussen, S. (2000). Ruin Probabilities. World Scientific, River Edge, NJ.

Fichtenholz, G. M. (1969). Differential and Integral Calculus, Vol. 2. Fizmatgiz, Moscow (in Russian).

Gerber, H. U. (1979). An Introduction to Mathematical Risk Theory. Huebner, Philadelphia, PA.

Gerber, H. U. (1988). Mathematical fun with ruin theory. Insurance Math. Econom. 7, 15-23.

Gerber, H. U. (1989). From the convolution of uniform distributions to the probability of ruin. Schweiz. Verein. Versicherungsmath. Mitt. 1989, 283-292.

Grandell, J. (1991). Aspects of Risk Theory. Springer, New York.

Ignatov, Z. G. AND Kaishev, V. K. (2000). Two-sided bounds for the finite time probability of ruin. Scand. Actuarial J. 2000, 46-62.

Ignatov, Z. G., Kaishev, V. K. And Krachunov, R. S. (2001). An improved finite-time ruin probability formula and its Mathematica implementation. Insurance Math. Econom. 29, 375-386.

Picard, P. ANd Lefèvre, C. (2001). On the probability of (non-) ruin in infinite time. Scand. Actuarial J. 2001, 148-161.

Shiu, E. S. W. (1987). Convolution of uniform distributions and ruin probability. Scand. Actuarial J. 1987, $191-197$.

ShiU, E. S. W. (1989). Ruin probability by operational calculus. Insurance Math. Econom. 8, 243-249. 